\title{
MODELO MATEMÁTICO PARA PREVISÃO DA TEMPERATURA DA SOJA ARMAZENADA EM SILOS
}

\author{
Marcos Flávio Pinto Moreira ${ }^{1}$ \\ Ronaldo Guimarães Corrêa ${ }^{2}$ \\ José Teixeira Freire ${ }^{3}$
}

\begin{abstract}
Resumo: A soja (Glycine Max) é um dos grãos mais exportados pelo Brasil e elevadas temperaturas durante o seu armazenamento acarretam a deterioração dos grãos, sendo, por isso, de fundamental importância o estudo da transferência de calor. O objetivo deste trabalho foi propor e validar um modelo matemático bidimensional para prever a temperatura em um silo de armazenagem de soja. O fenômeno da radiação solar foi incluído na modelagem de maneira detalhada. O modelo matemático foi resolvido utilizando o método de diferenças finitas. A validação do modelo foi feita a partir de temperaturas obtidas em um silo experimental. O erro médio entre as temperaturas calculadas e experimentais foi menor do que 6,3\%. Previsões da umidade dos grãos foram feitas a partir da modelagem proposta e mostraram-se em acordo com a literatura.
\end{abstract}

Palavras-chave: soja, silos, transferência de calor, umidade.

\begin{abstract}
Soybean (Glycine Max) is one of the largest Brazilian agricultural exports and, as generally high storage temperature is a factor of soybean deterioration, it is very important to investigate heat transfer in silos. The objective of this research was to propose and validate a two dimensional mathematical model based on the continuous theory to describe the temperature distribution inside a soybean silo. The phenomenon of solar radiation was included in the modeling in detail. The mathematical model was solved using the finite differences method. Model validation was made from experimental temperatures obtained in a model soybean silo. The average error between calculated and measured temperatures inside the silo was lower than $6.3 \%$. Moisture content of grains was predicted from the proposed modeling and the obtained estimates were in accord to literature.
\end{abstract}

Keywords: soybean, silos, heat transfer, moisture content.

\footnotetext{
${ }^{1}$ Universidade Estadual do Oeste do Paraná. E-mail: marcosfpmoreira@gmail.com

${ }^{2}$ Departamento de Engenharia Química, Universidade Federal de São Carlos. E-mail: ronaldo@ufscar.br

${ }^{3}$ Departamento de Engenharia Química, Universidade Federal de São Carlos. E-mail: freire@ufscar.br
} 


\section{INTRODUÇÃO}

A soja é uma planta arbustiva da família das leguminosas - nome científico: Glycine Max (L.) Merril domesticada pelos chineses na Ásia Central há cerca de 5 mil anos. Ao lado do arroz, do milho e do trigo é uma das principais lavouras do planeta, com produção anual superior a 100 milhões de toneladas e o Brasil está entre os maiores produtores mundiais (Hasse, 1996).

Vários produtos podem ser obtidos a partir da soja, tais como: produtos de soja integral, a proteína crua (uso comestível, uso industrial e farelo) e o óleo cru, a partir do qual podem ser obtidos esteróis, ácidos graxos, glicerol, lecitina e o óleo refinado (Hasse, 1996). O óleo refinado, em especial, tem uso técnico e uso comestível; é utilizado na cozinha e também pode dar origem à gordura hidrogenada que é utilizada na indústria alimentícia para a fabricação de bolachas, margarinas, sorvetes, chocolates, etc.

Estima-se que $30 \%$ da produção agrícola brasileira seja perdida na lavoura, na colheita, no transporte e na armazenagem (Lorini et al., 2002). As maiores perdas ocorrem na armazenagem, pois a duração dessa etapa é a mais longa do processo.

Entre os fatores que mais influenciam a qualidade de grãos e sementes armazenados em silos estão a ação de fungos, ácaros e insetos, os danos mecânicos e a umidade e temperatura da massa de grãos (Puzzi, 1986; Carvalho e Nakagawa, 1980).

O controle da temperatura em um silo é de grande importância, pois temperaturas acima de $42^{\circ} \mathrm{C}$ deterioram os grãos (Silva, 1998). Insetos também causam perdas dos grãos armazenados em temperaturas na faixa de 23 a $35^{\circ} \mathrm{C}$ e umidade dos grãos de 12 a $15 \%$ (base úmida (Puzzi, 1986; Carvalho e Nakagawa, 1980).

A temperatura na massa de grãos é afetada por fontes externas de calor (radiação solar direta, radiação difusa e transferência de calor por convecção com o ar ambiente) e fontes internas. As fontes internas são os calores de respiração do grão, dos insetos, ácaros e fungos (Jia et al., 2000). Segundo Christensen et al. (1969) apud Muir (1970) insetos e fungos são as principais fontes de geração de calor interna na massa de grãos.

Quanto ao transporte de calor na massa de grãos, Smith \& Sokhansanj (1990b) verificaram que a convecção natural não é significante para grãos de tamanho reduzido, como no caso do trigo. $\mathrm{O}$ processo dominante de transferência de calor na massa de grãos é a condução.

Desenvolver um modelo matemático que seja capaz de prever a temperatura dentro de um silo armazenador de grãos é algo de fundamental importância no objetivo da manutenção da qualidade dos grãos. A partir de um modelo confiável é possível prever a temperatura na massa de grãos e com isso estabelecer uma estratégia de aeração adequada, o que pode reduzir a necessidade de tratamento químico para o controle de insetos, ácaros e fungos (Jia et al., 2001).

A literatura apresenta diversos trabalhos que estudam a transferência de calor na armazenagem de grãos em silos verticais e em formato cilíndrico. Os grãos mais largamente utilizados nos estudos são o trigo (Muir, 1970; Converse et al., 1973; Yaciuk et al. 1975; Smith \&Sokhansanj, 1990a; Jia et al., 2000a; Jia et al., 2001; Jian et al., 2009; Stenning, 2012), o arroz (Abe \& Basunia, 1996; Jia et al., 2000b; Iguaz et al., 2004; Prachayawarakorn et al., 2005) e o milho (Dona \& Stewart, 1988; Fohr \& Moussa, 1994; Andrade et al., 2004). Diferentes modelos matemáticos são propostos, com uma dimensão (Muir, 1970; Yaciuk et al. 1975), duas dimensões (Dona \& Stewart, 1988; Smith \&Sokhansanj, 1990a; Abe \& Basunia, 1996; Jia et al., 2000ab; Jia et al., 2001; Iguaz et al., 2004; Rodríguez et al., 2011; Stenning, 2012) e três dimensões (Alagusundaram et al., 1990; Andrade et al., 2004; Jian et al., 2005). Diferentes condições de contorno são propostas, mas poucos são os trabalhos que se referem ao mesmo tempo a condições convectivas e radiativas nas paredes do silo (Yaciuk et al. 1975; Jia et al., 2000b; Jia et al., 2001). As equações resultantes são resolvidas por métodos como: diferenças finitas (Muir, 
1970; Yaciuk et al., 1975; Dona \& Stewart, 1988; Abe \& Basunia, 1996; Iguaz et al., 2004; Stenning, 2012), elementos finitos (Smith \&Sokhansanj, 1990a; Jia et al., 2000ab; Jia et al., 2001), colocação ortogonal (Rodríguez et al., 2011) e elementos discretos (Rusinek \& Kobilka, 2014). Para a validação da modelagem, alguns trabalhos se utilizam de dados experimentais gerados a partir de silos reais e outros se utilizam de dados obtidos a partir de silos experimentais. $\mathrm{O}$ diâmetro e a altura dos silos podem variar de dezenas de centímetros (Beukema et al., 1983; Abe \& Basunia, 1996) a alguns metros (Jia et al., 2000b). Quanto ao tempo de armazenamento avaliado, varia de horas (Jia et al., 2000a) a dezenas de meses (Jian et al, 2009).

Apesar dos estudos já realizados, existe ainda uma carência de informações com relação à soja. Além disso, a modelagem matemática pode ser melhorada principalmente no que diz respeito ao tratamento das condições de contorno relativas à radiação. Dessa forma os objetivos deste trabalho são:

a) desenvolver uma modelagem matemática para prever a temperatura em um silo de armazenagem de soja que inclua de maneira detalhada a influência da radiação sobre o fenômeno levando em conta a movimentação solar aparente,

b) validar o modelo com dados obtidos experimentalmente, $\mathrm{e}$

c) estimar a umidade na massa de grãos a partir da modelagem proposta.

\section{MATERIAIS E MÉTODOS}

\subsection{Desenvolvimento do modelo de transferência de calor}

Analisando a literatura é possível encontrar modelos que buscam incluir o maior número possível de fenômenos para obter uma boa previsão da temperatura (Smith \&Sokhansanj, 1990a; Fohr \& Moussa, 1994; Iguaz et al., 2004; Prachayawarakorn et al., 2005; Rodríguez et al., 2011). Sabe-se, no entanto, que alguns fenômenos podem ser desprezados em determinadas condições, simplificando assim a modelagem. Segundo Smith \& Sokhansanj (1990b), a convecção natural não é significante para grãos de tamanho reduzido, o que foi verificado no caso do trigo. A permeabilidade do meio poroso para grãos pequenos torna-se muito baixa devido ao reduzido tamanho dos poros, o que dificulta a movimentação do ar, fazendo com que a condução domine o processo de transferência. Dessa forma, o balanço de quantidade de movimento para a fase gasosa pode ser desprezado devido às baixas velocidades do ar. Segundo Smith \& Sokhansanj (1990a) a convecção natural apresenta influência significante sobre a temperatura apenas para valores do número de Rayleigh acima de 1.400 . Esses autores verificaram que mesmo um valor de 3.300 para o número de Rayleigh indica ainda pouca influência da convecção natural sobre a temperatura. Como neste trabalho o valor para o número de Rayleigh está em torno de 3.000, desprezou-se o efeito da convecção natural sobre a temperatura.

Outra suposição que pode ser feita nos estudos de transferência de calor em silos é a respeito da variação da umidade da massa de grãos. Sabe-se que a variação de umidade dos grãos é muito lenta perto da variação de temperatura, de modo que a variação de umidade pode ser desprezada. Prachayawarakorn et al. (2005) verificaram que a umidade na maior parte do silo ficou em torno de $14,5 \%$ (b.u.) e que apenas próximo da parede houve um aumento para $16 \%$ (b.u.), uma diferença de apenas $1,5 \%$ após 6 meses de armazenamento. Jian et al. (2009) também verificaram variações menores do que $1 \%$ após um ano de armazenamento. Apesar de se desconsiderar a variação de umidade na massa de grãos, ainda é possível prever tal variação ao se admitir equilíbrio de sorção local conforme Abe \& Basunia (1996) propuseram em seu estudo. No item 3.3 deste artigo apresenta-se uma previsão da umidade na massa de grãos seguindo esse método.

A respeito das fontes internas de geração de calor, sabe-se que o calor de 
respiração dos grãos é desprezível frente ao calor liberado pela ação de insetos, fungos e ácaros (Christensen et al., 1969 apud Muir, 1970). Considerando então o não desenvolvimento de fungos, ácaros e insetos na massa de grãos, assume-se como desprezível a geração de calor interna.

Considerando ainda meio poroso isotrópico e homogêneo, equilíbrio térmico local entre as fases sólida e fluida, propriedades físicas constantes, variação desprezível de temperatura na direção angular, chega-se ao seguinte balanço diferencial de energia:

$$
\frac{\partial \mathrm{T}}{\partial \mathrm{t}}=\alpha_{r}\left(\frac{\partial^{2} \mathrm{~T}}{\partial \mathrm{z}^{2}}+\frac{1}{\mathrm{r}} \frac{\partial \mathrm{T}}{\partial \mathrm{r}}+\frac{\partial^{2} \mathrm{~T}}{\partial \mathrm{r}^{2}}\right)
$$

onde $\alpha_{\mathrm{T}}$ é a difusividade térmica efetiva do meio, $\mathrm{T}$ a temperatura, $\mathrm{t}$ o tempo, $\mathrm{r}$ a coordenada radial e $\mathrm{z}$ a coordenada axial.

Alguns trabalhos assumem a Equação (1) como o balanço de energia a ser considerado no estudo (Abe \& Basunia, 1996; Jia et al., 2001. Stenning (2012) utiliza a Equação (1) considerando duas difusividades térmicas efetivas, uma radial e outra axial.

Assumiu-se como condição de simetria que:

em r=0, $\quad \frac{\partial \mathrm{T}}{\partial \mathrm{r}}=0$

Como condição inicial assumiu-se que em $\mathrm{t}=0$ :

$$
\mathrm{T}(\mathrm{r}, \mathrm{z})=\mathrm{T}_{\mathrm{o}}
$$

Como condições de contorno assumiu-se que a resistência convectiva à transferência de calor na parte interna do silo pode ser desprezada de forma que a temperatura na região de contorno é a própria temperatura da parede. Assumiuse ainda como desprezível a resistência condutiva à transferência de calor na parede do silo.

Para as temperaturas das paredes do silo (fundo, lateral e topo) foram propostos balanços de energia.

Considerou-se que no fundo do silo só há troca de calor convectiva e que nas paredes superior (topo) e lateral estão presentes a radiação solar direta e a radiação difusa $\left(\mathrm{q}_{\mathrm{rad}}\right)$, o calor radiativo recebido do meio ambiente (superfície terrestre e atmosfera), o calor radiativo emitido pela parede do silo e as trocas convectivas (Duffie e Beckman, 1974).

Como simplificação admitiu-se que a soma do calor emitido pela superfície terrestre e pelo céu é dada pela lei de Stefan-Boltzmann (Holman, 1983) onde o calor emitido é dado por " $\sigma \cdot \mathrm{T}_{\mathrm{amb}} 4$ ".

Os balanços de energia assim obtidos para as paredes do silo são dados por:

para $\mathrm{z}=0$ :

$$
\text { e. } \rho_{\text {silo }} \cdot C p_{\text {silo }} \frac{\mathrm{dT}_{\mathrm{p}}^{\text {findo }}}{\mathrm{dt}}=\mathrm{h}_{\text {ext-findo }}\left(\mathrm{T}_{\text {solo }}-\mathrm{T}_{\mathrm{p}}^{\text {findo }}\right)+\mathrm{h}_{\text {int-finuto }}\left(\mathrm{T}_{\mathrm{z}=0}-\mathrm{T}_{\mathrm{p}}^{\text {findo }}\right)
$$

para $\mathrm{Z}=\mathrm{L}$ :

$$
\begin{aligned}
\text { e. } \rho_{\text {silo }} \cdot \mathrm{Cp}_{\text {silo }} \frac{\mathrm{dT}_{\mathrm{p}}^{\text {topo }}}{\mathrm{dt}}=\mathrm{q}_{\mathrm{rad}}^{\text {topo }}+\mathrm{h}_{\text {ext-Lopo }} & \left(\mathrm{T}_{\text {amb }}-\mathrm{T}_{\mathrm{p}}^{\text {topo }}\right) \\
& -\varepsilon \cdot \sigma \cdot\left(\mathrm{T}_{\mathrm{p}}^{\text {topo }}\right)+\alpha \cdot \sigma \cdot\left(\mathrm{T}_{\text {amb }}^{4}\right)+\mathrm{h}_{\text {int-topo }}\left(\mathrm{T}_{\mathrm{z}=\mathrm{L}}-\mathrm{T}_{\mathrm{P}}^{\text {topo }}\right)
\end{aligned}
$$

para $\mathrm{r}=\mathrm{R}$ :

$$
\begin{aligned}
& \frac{\left[(\mathrm{R}+\mathrm{e})^{2}-\mathrm{R}^{2}\right]}{2(\mathrm{R}+\mathrm{e})} \cdot \rho_{\text {silo }} \cdot \mathrm{Cp}_{\text {silo }} \frac{\mathrm{dT}_{\mathrm{p}}^{\mathrm{bat}}}{\mathrm{dt}}=\mathrm{q}_{\mathrm{rad}}^{\mathrm{lat}}+\mathrm{h}_{\text {ext-lat }}\left(\mathrm{T}_{\mathrm{amb}}-\mathrm{T}_{\mathrm{p}}^{\mathrm{hat}}\right) \\
& -\varepsilon \cdot \sigma \cdot\left(\mathrm{T}_{\mathrm{p}}^{\mathrm{hat}}\right)+\alpha \cdot \sigma \cdot\left(\mathrm{T}_{\mathrm{amb}}^{4}\right)+[\mathrm{R} /(\mathrm{R}+\mathrm{e})] \cdot \mathrm{h}_{\mathrm{int}-\mathrm{Lat}}\left(\mathrm{T}_{\mathrm{r}=\mathrm{R}}-\mathrm{T}_{\mathrm{p}}^{\mathrm{at}}\right)
\end{aligned}
$$


Cabe salientar que nas Equações (46) a última parcela do lado direito é nula devido às condições de contorno impostas.

A difusividade térmica efetiva $\left(\alpha_{\mathrm{T}}\right)$ foi tomada constante com o valor de $1,12 \times 10^{-7} \mathrm{~m}^{2} \cdot \mathrm{s}^{-1}$ (Moreira e Freire, 2004).

Nas equações acima "e" é a espessura da parede do silo e $\mathrm{R}$ o raio interno do silo. Considerou-se o silo como sendo um cilindro perfeito. A massa específica da parede do silo $\left(\rho_{\text {silo }}\right)$ foi assumida como $7.800 \mathrm{~kg} \cdot \mathrm{m}^{-3}$ (Holman, 1983) e o $\mathrm{Cp}$ do silo $\left(\mathrm{Cp}_{\text {silo }}\right)$ como 480 J.kg ${ }^{-1} \cdot \mathrm{K}^{-1}$ (Holman, 1983). O coeficiente de troca térmica externo $\left(\mathrm{h}_{\mathrm{ext}}\right)$ foi considerado constante com valor igual a $12 \mathrm{~W} \cdot \mathrm{m}^{-2} \cdot \mathrm{K}^{-1}$ (Kreith, 1973).

A temperatura ambiente $\left(\mathrm{T}_{\mathrm{amb}}\right)$ e a temperatura do solo abaixo do silo $\left(\mathrm{T}_{\text {solo }}\right)$ foram medidas durante o experimento.

A emissividade $(\varepsilon)$ e a absortividade $(\alpha)$ da parede do silo, por razões de simplificação, foram assumidas constantes para toda a faixa de comprimento de onda com valores de 0,28 e 0,37 respectivamente (Andrade et al., 2004; Holman, 1983). A variável " $\sigma$ " representa a constante de Stefan-Boltzmann com valor de $5,67 \cdot 10^{-8} \mathrm{~W} \cdot \mathrm{m}^{-2} \cdot \mathrm{K}^{-4}$.

Os calores de radiação (direta e difusa) por unidade de área absorvidos pelo topo $\left(\mathrm{q}_{\mathrm{rad}}{ }^{\text {topo }}\right)$ e pela parede lateral do silo $\left(\mathrm{q}_{\mathrm{rad}}^{\text {lat }}\right)$ são dados respectivamente por:

$$
\begin{gathered}
\mathrm{q}_{\text {rad }}^{\text {topo }}=\alpha \cdot \mathrm{q}_{\text {н }} \\
\mathrm{q}_{\text {rad }}^{\text {lat }}=\alpha \alpha \cdot \mathrm{q}_{\mathrm{v}}
\end{gathered}
$$

onde $\alpha_{\text {lat }}$ é a porcentagem da área lateral que é atingida pela radiação solar direta e tem o valor de $1 / \pi$.

Os valores para o calor de radiação (direta e difusa) horizontal $\left(\mathrm{q}_{\mathrm{H}}\right)$ foram tomados como sendo os valores para a cidade de Campinas (SP-Brasil, 22 $2^{\circ} 52^{\prime} \mathrm{S}$, $47^{\circ} 02^{\prime} \mathrm{W}, 685 \mathrm{~m}$ acima do nível do mar) que fica próxima à cidade de São Carlos onde estava localizado o silo experimental (SP-Brasil, 21 ${ }^{\circ} 58^{\prime} \mathrm{S}, 47^{\circ} 52^{\prime} \mathrm{W}, 829 \mathrm{~m}$ acima do nível do mar). Os valores para os calores de radiação estavam disponíveis na página da internet da Embrapa (Embrapa, 2005). Obter dados meteorológicos de um local próximo também foi o procedimento adotado por Muir (1970) para suprir a falta de tais informações.

Como simplificação considerou-se que a radiação difusa equivale a $10 \%$ da radiação solar direta horizontal $\left(q_{\text {dif }}=0,1 . q_{H}{ }^{\text {dir }}\right)$, o que significa considerar céu claro (Kreith, 1973), consideração essa que está bem próxima da realidade visto que o período avaliado apresentou céu claro na grande maioria dos dias. Assim tem-se que:

$$
\mathrm{q}_{\mathrm{H}}=\mathrm{q}_{\mathrm{H}}^{\text {dir }}+\mathrm{q}_{\text {dif }}=1,1 \cdot \mathrm{q}_{\text {н }}^{\text {dir }}
$$

Com os valores para o calor de radiação direta horizontal $\left(\mathrm{q}_{\mathrm{H}}{ }^{\mathrm{dir}}\right)$ foi possível obter os valores do calor de radiação direta que atinge a parede lateral do silo $\left(\mathrm{qv}^{\mathrm{dir}}\right)$ através da seguinte equação (Kreith, 1973):

$$
\mathrm{q}_{\mathrm{v}}^{\mathrm{dir}}=\mathrm{q}_{\mathrm{H}}^{\mathrm{dir}} \cdot \tan (\mathrm{zen})
$$

onde zen é a distância zenital.

Segundo Filho e Saraiva (2004) a distância zenital é dada por:

$$
\begin{aligned}
\text { zen }=\operatorname{acos}[ & \operatorname{sen} \phi \cdot \operatorname{sen} \delta+ \\
& \left.+\cos \phi \cdot \cos \delta \cdot \cos \mathrm{H}_{\mathrm{v}}\right]
\end{aligned}
$$

onde $\phi$ é a latitude do lugar (para o lugar onde está o silo em São Carlos é igual a $21^{\circ} 58^{\prime}$ ) e $\delta$ é a declinação solar e pode ser dada por (Cooper, 1969):

$$
\delta=23,45^{\circ} \cdot \operatorname{sen}\left[360^{\circ} \frac{(284+\mathrm{n})}{365}\right]
$$

sendo $\mathrm{n}$ o dia Juliano $\left(\mathrm{n}=1\right.$ em $1^{\mathrm{o}}$ de janeiro e $n=365$ em 31 de dezembro).

O ângulo horário do Sol verdadeiro $\left(\mathrm{H}_{\mathrm{V}}\right)$ é dado por (Filho e Saraiva, 2004):

$$
\mathrm{H}_{\mathrm{v}}=\mathrm{H}_{\mathrm{s}}-\mathrm{ET}
$$

onde $\mathrm{H}_{\mathrm{M}}$ é o ângulo horário do Sol médio em São Carlos. Como a longitude do lugar onde está o silo em São Carlos é de $47^{\circ} 52^{\prime}$ oeste, o local onde está o silo tem uma longitude que a coloca $2^{\circ} 52^{\prime}$ a oeste do 
meridiano central do fuso horário local que é de $45^{\circ}$ oeste. A hora solar média em São Carlos está então atrasada com relação à hora do relógio $\left(\mathrm{H}_{\mathrm{L}}=\right.$ hora legal $)$ por $2^{\circ} 52^{\prime} / 15^{\circ}=0,19111 \mathrm{~h}=11,5 \mathrm{~min}$. Assim o ângulo horário do Sol médio no local onde está o silo é dado por:

$$
\mathrm{H}_{\mathrm{M}}=\mathrm{H}_{\mathrm{L}}-0,19-12
$$

$O$ valor de ET representa a Equação do Tempo (indiretamente relativa à "Ephemeris Transit") e relaciona o ângulo horário do Sol médio com o ângulo horário do Sol verdadeiro, pois a passagem sucessiva do Sol por um mesmo meridiano leva um pouco menos que $24 \mathrm{~h}$. Os valores de -ET foram calculados com o auxílio do programa "aa-56" (Moshier, 2005).

A Figura 1 apresenta os valores obtidos para -ET para cada um dos dias simulados.

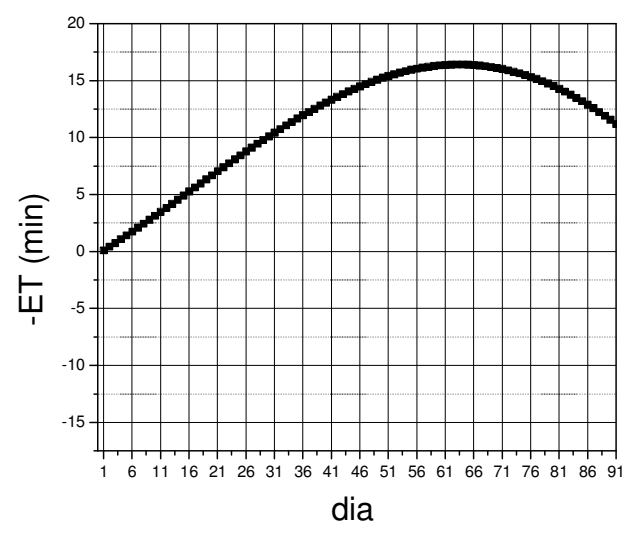

Figura 1. Valores de -ET em função do dia $\left(\right.$ dia $=1$ equivale a $1^{\circ}$ de setembro de 2005).

Os valores para o calor de radiação (direta e difusa) na parede lateral ( $\left.\mathrm{q}_{\mathrm{v}}\right)$ foram obtidos pela soma da radiação difusa $\left(\mathrm{q}_{\text {diff }}\right)$ com a radiação direta na lateral $\left(\mathrm{qv}^{\mathrm{dir}}\right)$ :

$$
\mathrm{q}_{\mathrm{v}}=\mathrm{q}_{\mathrm{v}}^{\mathrm{dir}}+\mathrm{q}_{\text {dif }}
$$

\subsection{Solução numérica do modelo de transferência de calor}

Para a resolução do modelo e obtenção dos valores calculados de temperatura em função do tempo utilizou- se o Método das Diferenças Finitas (MDF) com diferença central. A malha foi composta por 117 pontos no total (9 na direção radial x 13 na direção axial). Verificou-se que acima deste número de pontos não ocorriam mudanças nos valores calculados das temperaturas. $\mathrm{O}$ conjunto de equações diferenciais ordinárias foi integrado no tempo pelo método de Runge-Kutta de quarta ordem utilizando-se o software Matlab® para a resolução do problema.

\subsection{Descrição do aparato experimental utilizado para a validação do modelo}

Para a obtenção dos dados experimentais de temperatura foi construído um silo em escala reduzida a fim de diminuir os custos da experimentação. A Figura 2 apresenta um esquema do aparato experimental.

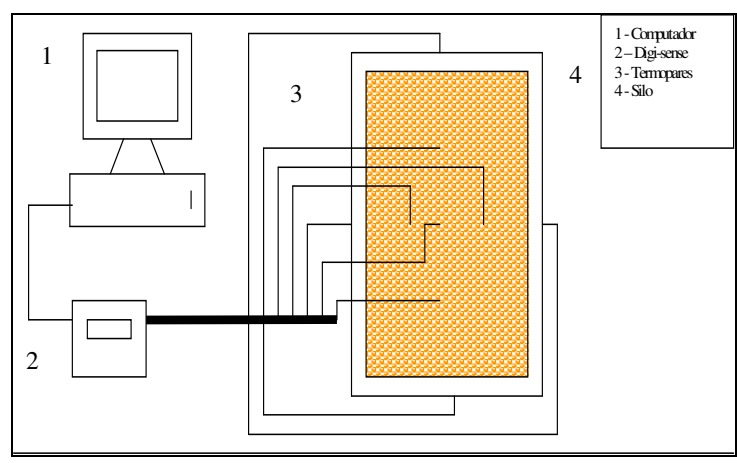

Figura 2. Esquema do aparato

experimental utilizado para a obtenção das temperaturas experimentais.

O silo foi feito com uma chapa de aço inoxidável de $2 \mathrm{~mm}$ de espessura em formato cilíndrico, com $59 \mathrm{~cm}$ de altura (H) e $38 \mathrm{~cm}$ de diâmetro (2R) aproximadamente. $\mathrm{O}$ silo foi completamente cheio de soja e ficou fixado em uma base de concreto. A temperatura inicial da soja em toda massa de grãos era de $29^{\circ} \mathrm{C}$ com umidade de $12 \%$ (base seca).

As temperaturas foram medidas nos meses de setembro, outubro e novembro de 2005 com termopares do tipo $\mathrm{T}$ (Cobre-Constantan) que estavam conectados a um receptor (Digi-sense, Scanning Thermocouple Thermometer modelo 21800-00A Cole Parmer) que por 
sua vez estava conectado a um computador por meio de uma interface RS-232 para o monitoramento contínuo das temperaturas. Foram monitoradas as temperaturas nos pontos mostrados na Figura 2. Foram eles:

a) em $\mathrm{r}=0$ e $\mathrm{z}=\mathrm{H} / 4, \mathrm{H} / 2$ e $3 \mathrm{H} / 4$;

b) em $\mathrm{r}=\mathrm{R} / 2$ e $\mathrm{z}=\mathrm{H} / 2$ e $\theta=0^{\circ}$ (norte), $90^{\circ}$ (leste), $180^{\circ}$ (sul), $270^{\circ}$ (oeste);

c) na parte externa da parede lateral do silo em $\mathrm{z}=\mathrm{H} / 2$ nas direções leste e oeste;

d) na parte externa da parede superior $(\mathrm{r}=0 ; \mathrm{z}=\mathrm{H}), \mathrm{e}$

e) na parte externa da parede inferior $(r=0$; $\mathrm{z}=0$ ).

\section{RESULTADOS E DISCUSSÕES}

\section{$3.1 \quad$ Valores experimentalmente}

obtidos

Em relação às temperaturas medidas na massa de grãos na posição central $(\mathrm{r}=0)$, a Figura 3 apresenta os resultados obtidos.

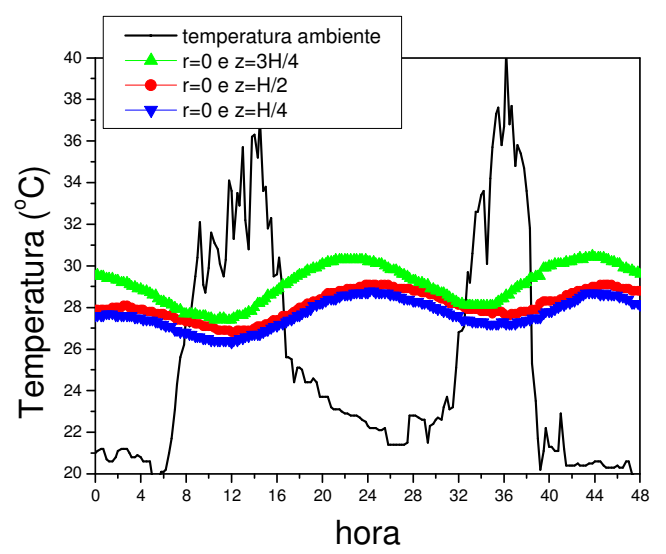

Figura 3. Temperaturas experimentais em função do tempo.

Os resultados apresentados na Figura 3 foram típicos para todo o período avaliado.

Através da Figura 3 verificou-se um comportamento oscilatório tanto da temperatura ambiente quanto da temperatura na massa de grãos. $O$ comportamento oscilatório da temperatura ambiente se deve em parte à variação da radiação solar que atinge a atmosfera, variação essa provocada pelo movimento de rotação terrestre.

Através da Figura 3 verificou-se ainda que a variação da temperatura na massa de grãos foi menor que a da temperatura ambiente. Enquanto na massa de grãos a temperatura variou entre $26 \mathrm{e}$ $30^{\circ} \mathrm{C}$, no ambiente a temperatura variou entre 20 e $40^{\circ} \mathrm{C}$. Além disso, verificou-se que, para o momento em que a temperatura ambiente esteve em torno de seu valor máximo, a temperatura na massa de grãos esteve em torno de seu valor mínimo. Tanto a diferença de variação de temperatura quanto $\mathrm{o}$ deslocamento temporal dos valores máximos e mínimos das temperaturas na massa de grãos e da temperatura ambiente foram ocasionados pelas resistências térmicas presentes entre o ambiente e o centro do silo, como por exemplo, o inverso da condutividade térmica efetiva do meio poroso e a distância entre a parede do silo e o seu centro (distância radial).

Em relação à influência da posição axial sobre a temperatura, verificou-se que, do fundo para a superfície da massa de grãos, houve um aumento da temperatura. Esse resultado também foi obtido por Dona \& Stewart (1988) na simulação feita para o armazenamento de milho. As diferenças foram maiores durante a noite, momento em que a temperatura ambiente se encaminhava para o valor mínimo. Já as menores diferenças entre as temperaturas ocorreram por volta do meio-dia, momento em que a temperatura ambiente se encaminhava para o máximo.

Para a posição $\mathrm{r}=\mathrm{R} / 2$ e $\mathrm{z}=\mathrm{H} / 2$, foram medidas as temperaturas em quatro posições angulares na massa de grãos, uma na direção norte, outra na direção leste, outra na direção sul e a quarta na direção oeste. A Figura 4 apresenta os resultados obtidos, os quais foram típicos para todo o período avaliado.

Em relação à influência da posição angular, verificou-se, como já era esperado, uma diferença entre as temperaturas nas posições "leste", "norte", "oeste" e "sul". Isso ocorreu devido ao movimento aparente do Sol em torno do silo, ou seja, devido à rotação terrestre. Como o Sol nasce no leste, a incidência da radiação solar começa nessa direção fazendo com que a massa de grãos tenha 
sua temperatura elevada também nesse local.

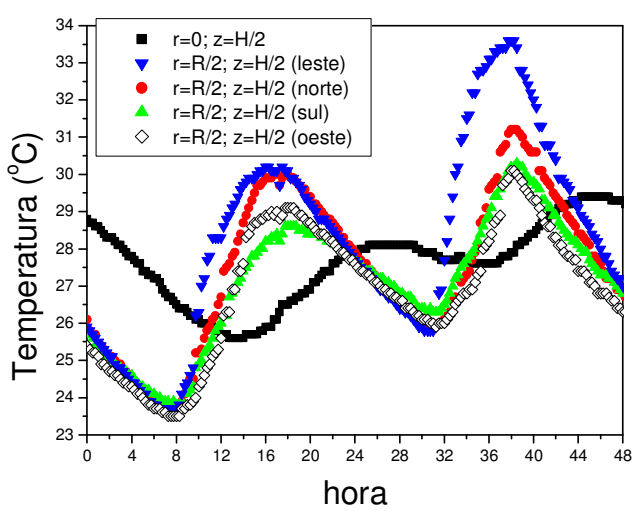

Figura 4. Temperaturas experimentais na posição $r=R / 2$ e $z=H / 2$ em função do tempo.

Devido à distância zenital característica do local onde estava o silo no período avaliado (primavera), os raios solares, após incidirem na parede leste do silo, se encaminhavam para a parede norte e por fim à parede oeste. Esse fenômeno ficou traduzido nas temperaturas medidas na massa de grãos como se verificou através da análise da Figura 4. Jian et al. (2009), em estudo com o trigo, também verificaram um comportamento semelhante da temperatura em função da posição angular.

Com relação à influência da distância radial, verificou-se que à medida que a distância radial aumentou, ou seja, do centro em direção à parede, a massa de grãos apresentou maior variação de temperatura como ficou verificado na Figura 4. Enquanto no centro houve uma variação de $2^{\circ} \mathrm{C}$, na posição de $\mathrm{r}=9,5 \mathrm{~cm}$ a variação foi de $7^{\circ} \mathrm{C}$.

Em relação às temperaturas medidas nas paredes do silo, a Figura 5 apresenta os resultados obtidos. Tais resultados são típicos para todo o período avaliado.

Verificou-se que foi o topo do silo que apresentou os maiores valores de temperatura no período avaliado (primavera), seguido da parede lateral, do ambiente e do fundo do silo. Caso os experimentos tivessem sido realizados durante o inverno, quando a distância zenital é maior, a temperatura no topo provavelmente não teria atingido valores tão diferentes das temperaturas na lateral do silo. Yaciuk et al. (1975) verificaram em suas simulações que o uso de tinta branca nas paredes externas do silo poderia diminuir a temperatura das paredes e, por conseguinte, da massa de grãos.

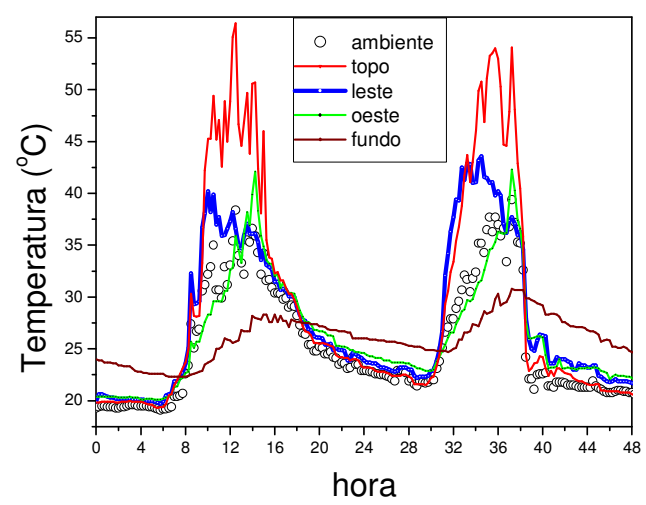

Figura 5. Temperaturas experimentais na posição $\mathrm{r}=\mathrm{R}$ e $\mathrm{z}=\mathrm{H} / 2$ (parede lateral) em função do tempo.

Em relação às temperaturas medidas na parede lateral do silo nas direções leste e oeste, foi possível verificar um comportamento deslocado das curvas de temperatura devido ao movimento aparente do Sol. Enquanto a parede leste atingia a temperatura máxima em torno de $10 \mathrm{~h}$ da manhã, a temperatura na parede oeste atingia seu valor máximo 4 horas depois.

Quando o Sol se pôs, as temperaturas no topo e na lateral do silo tenderam à temperatura do ambiente e durante a noite a temperatura no fundo do silo foi maior que as temperaturas do topo, da lateral e do ambiente.

\subsection{Validação da modelagem proposta}

A seguir são apresentadas algumas comparações entre as temperaturas medidas e calculadas para parte do mês de outubro/2005, do dia vinte (20) de outubro ao dia trinta e um (31) de outubro. Os resultados apresentados a seguir são típicos para todo o período avaliado.

Verifica-se através das Figuras 6 a 10 que os valores calculados de temperatura estão muito próximos dos 
valores experimentais para todas as posições avaliadas, tanto dentro do silo como em sua estrutura (parede de aço).

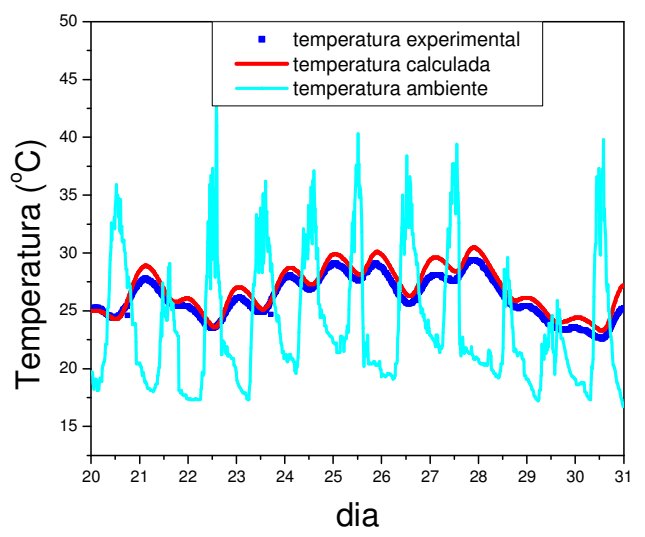

Figura 6. Temperaturas experimental e calculada para a posição $\mathrm{r}=0 \mathrm{e} \mathrm{z}=\mathrm{H} / 2 \mathrm{em}$ função do tempo.

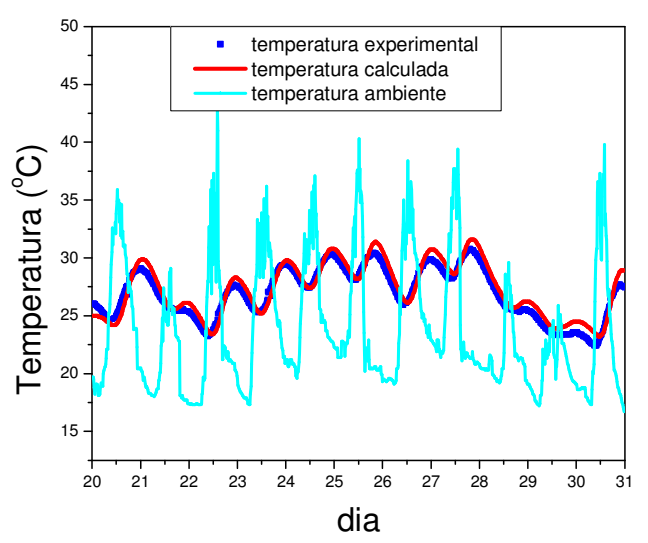

Figura 7. Temperaturas experimental e calculada para a posição $r=0$ e $z=3 \mathrm{H} / 4 \mathrm{em}$ função do tempo.

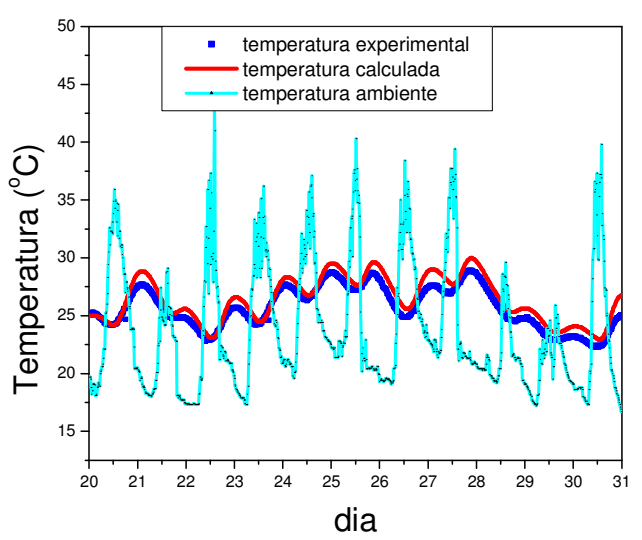

Figura 8. Temperaturas experimental e calculada para a posição $\mathrm{r}=0 \mathrm{e} \mathrm{z}=\mathrm{H} / 4 \mathrm{em}$ função do tempo.

A fim de quantificar a diferença entre os valores calculados e experimentais foram calculados os erros absolutos médios relativos de temperatura através da seguinte equação:

$$
\mathrm{E} \%=\frac{100}{\mathrm{~N}} \cdot \sum_{\mathrm{i}=1}^{N}\left[\frac{\left|\mathrm{T}_{\text {exp.i }}-\mathrm{T}_{\text {cadi. }}\right|}{\mathrm{T}_{\mathrm{exp}, \mathrm{i}}}\right]
$$

onde a temperatura foi utilizada com a unidade de graus Celsius. Cabe salientar que a Equação (16) só se aplica quando todas as temperaturas são maiores que zero graus Celsius, como aconteceu neste trabalho.

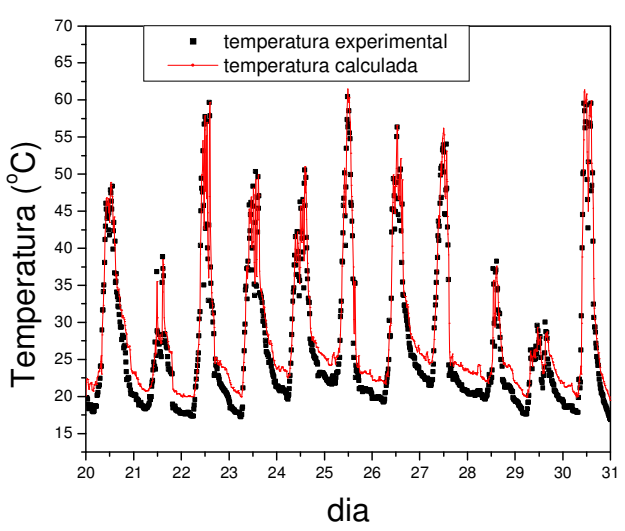

Figura 9. Temperaturas experimental e calculada para a parede superior do silo em função do tempo.

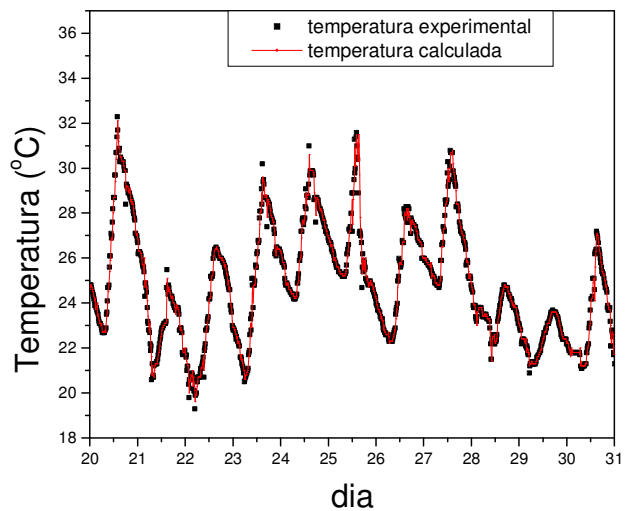

Figura 10. Temperaturas experimental e calculada para a parede do fundo do silo em função do tempo.

A Tabela 1 apresenta os erros para todo o período estudado.

Analisando a Tabela 1 pôde se verificar que os menores erros de temperatura na massa de grãos são obtidos 
nas medições feitas em $\mathrm{r}=0$, obtendo-se valores de 2,3 a 5,9\%. Em termos de temperatura, o maior erro obtido entre as temperaturas experimentais e calculadas foi de $1,8^{\circ} \mathrm{C}$. Yaciuk et al. (1975) obtiveram um erro máximo de $3{ }^{\circ} \mathrm{C}$ trabalhando com trigo, enquanto Abe \& Basunia (1996) obtiveram $1,82^{\circ} \mathrm{C}$ para o arroz.

Tabela 1. Erros mensais de temperatura.

\begin{tabular}{|l|c|c|c|}
\hline & Set & Out & Nov \\
\hline$(\mathrm{r}=0 ; \mathrm{z}=\mathrm{H} / 2)$ & $5,9 \%$ & $2,7 \%$ & $3,0 \%$ \\
\hline$(\mathrm{r}=0 ; \mathrm{z}=3 \mathrm{H} / 4)$ & $3,1 \%$ & $2,9 \%$ & $2,3 \%$ \\
\hline$(\mathrm{r}=0 ; \mathrm{z}=\mathrm{H} / 4)$ & $4,5 \%$ & $4,5 \%$ & $2,9 \%$ \\
\hline $\begin{array}{l}(\mathrm{r}=\mathrm{R} / 2 ; \mathrm{z}=\mathrm{H} / 2) \\
\text { média }\end{array}$ & $6,3 \%$ & $5,5 \%$ & $5,8 \%$ \\
\hline parede superior & $10,5 \%$ & $10,8 \%$ & $11,7 \%$ \\
\hline $\begin{array}{l}\text { parede lateral } \\
\text { (média) }\end{array}$ & $6,7 \%$ & $7,3 \%$ & $7,7 \%$ \\
\hline parede inferior & $1,2 \%$ & $0,8 \%$ & $0,9 \%$ \\
\hline
\end{tabular}

Em relação às temperaturas das paredes superior, lateral e inferior do silo, verificou-se que a parede inferior apresentou os menores erros, em torno de $1 \%$. De uma forma geral, a maioria dos erros ficaram abaixo de 7,7\%. Apenas na parede superior do silo foram maiores, em torno de $11 \%$. Isso ocorreu, pois a qualidade do ajuste (veja a Figura 9) não foi boa nas baixas temperaturas $\left(\sim 20^{\circ} \mathrm{C}\right)$. Deve-se lembrar que erros de $11 \%$ em temperaturas de $20^{\circ} \mathrm{C}$ significam $2,2^{\circ} \mathrm{C}$ de diferença entre os valores calculados e experimentais. Esse valor é muito pequeno em relação à faixa de temperatura na parede superior do silo em um único dia que foi de $15^{\circ} \mathrm{C}$ a $60^{\circ} \mathrm{C}$. Além disso, os erros de 10,5\% a $11,7 \%$ encontrados para a parede superior do silo não se repetiram para a massa de grãos, a qual apresentou valores menores que $6,3 \%$.

Como a modelagem proposta não incorporou a variável angular, foi feita a média aritmética das temperaturas angulares experimentais para a comparação com os valores calculados. As Figuras 11 e 12 apresentam essas comparações para a massa de grãos e para a parede lateral respectivamente.

Verificou-se que, apesar do movimento aparente do Sol em torno do silo, a temperatura média em $r=R / 2$ e $\mathrm{z}=\mathrm{H} / 2$ é satisfatoriamente prevista pelo modelo proposto. Os erros da temperatura calculada em relação à média experimental variaram de 5,8 a $6,3 \%$ na massa de grãos e de 6,7 a $7,7 \%$ na parede lateral. Jia et al. (2001) também aplicaram um modelo bidimensional sem geração de calor para prever a temperatura em um silo com 4,89m de diâmetro e $6,5 \mathrm{~m}$ de altura para a armazenagem de trigo e também obtiveram bons resultados.

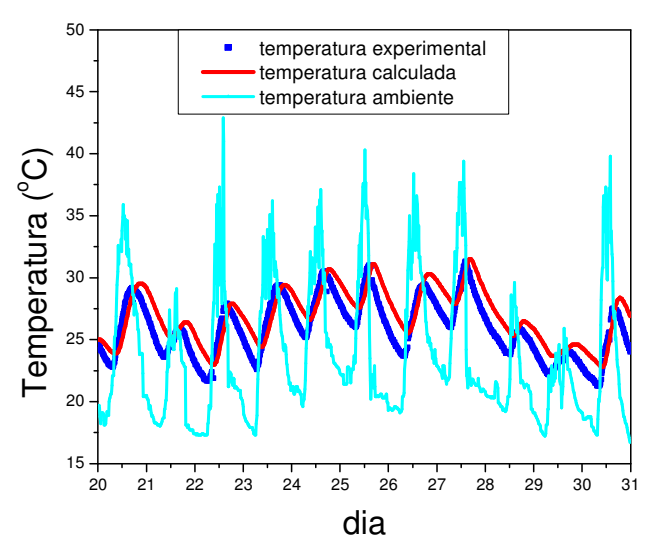

Figura 11. Temperaturas experimental (média angular) e calculada para a posição $\mathrm{r}=\mathrm{R} / 2$ e $\mathrm{z}=\mathrm{H} / 2$ em função do tempo (de 20 a 31 de outubro/2005).

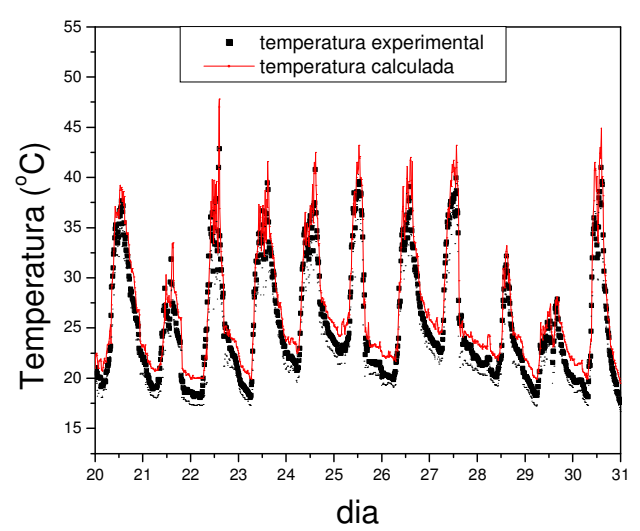

Figura 12. Temperaturas experimental (média angular) e calculada para a posição $\mathrm{r}=\mathrm{R}$ e $\mathrm{z}=\mathrm{H} / 2$ (parede lateral) em função do

tempo (de 20 a 31 de outubro/2005).

De maneira geral verificou-se que a modelagem proposta conseguiu obter resultados muito bons na previsão das temperaturas experimentais apesar de todas as simplificações propostas.

Acredita-se que os erros de 
temperatura obtidos para a massa de grãos e para as paredes do silo poderiam ter atingido valores ainda menores caso o calor de radiação horizontal $\left(\mathrm{q}_{\mathrm{H}}{ }^{\mathrm{dir}}\right.$ ) tivesse sido determinado experimentalmente.

É natural ainda esperar que a modelagem proposta neste trabalho sirva para prever a temperatura no armazenamento de outros grãos (como feijão, arroz, etc.) levando-se em conta, é claro, os novos valores como, por exemplo, o da difusividade térmica efetiva.

\subsection{Simulação da umidade na massa de grãos}

Considerando o seguinte modelo, proposto por Finkler et al. (2013), para a umidade de equilíbrio da soja:

$$
X_{c}=\left[\frac{\exp (-0,0119 . T+6,586)}{-\ln (\mathrm{UR})}\right]^{\frac{1}{1.509}}
$$

e utilizando as temperaturas calculadas através da modelagem proposta neste trabalho foi possível estimar a umidade da massa de grãos.

$\mathrm{Na}$ Equação (17) a umidade relativa utilizada (UR) está em valores decimais e foi atribuído um valor de $60 \%$. A temperatura está em Kelvin e a umidade é dada em valores percentuais (base seca).

A Figura 13 apresenta os valores obtidos para a umidade da soja nas posições $(r=0 ; z=H / 2),(r=R / 2 ; z=H / 2)$ e $(\mathrm{r}=\mathrm{R} ; \mathrm{z}=\mathrm{H} / 2)$.

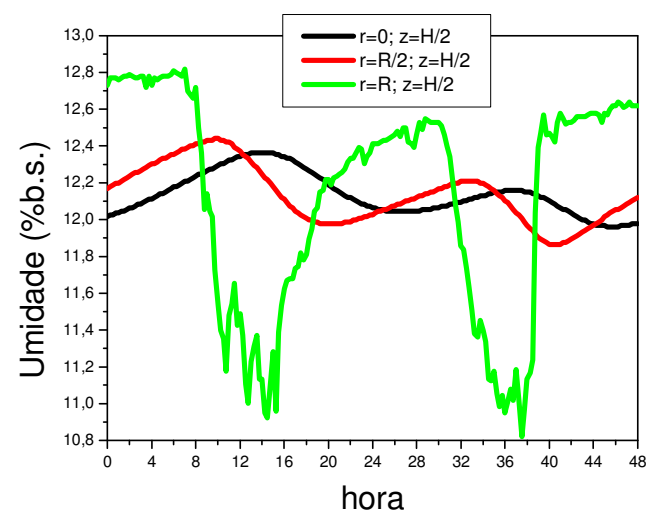

Figura 13. Umidades calculadas na massa de grãos em função do tempo.
Como era esperado, a maior variação de umidade ocorreu próximo à parede $(\mathrm{r}=\mathrm{R})$, cerca de $2 \%$, devido à maior variação de temperatura nesta posição. Abe \& Basunia (1996) verificaram variação experimental de umidade de $2 \%$, valor próximo aos verificados experimentalmente por Prachayawarakorn et al. (2005) e também por Jian et al. (2009).

\section{CONCLUSÕES}

Neste trabalho foi proposta e validada uma modelagem pseudohomogênea bidimensional para se prever a temperatura em um silo de armazenagem de soja. A validação foi realizada a partir de um silo experimental com $59 \mathrm{~cm}$ de altura e $38 \mathrm{~cm}$ de diâmetro.

Dentre outras hipóteses, a modelagem proposta desprezou a convecção natural, a migração de umidade e a geração de calor na massa de grãos. A modelagem levou em conta de forma detalhada o movimento solar e foi resolvida utilizando os métodos das diferenças finitas e de Runge-Kutta.

Verificou-se que a modelagem proposta conseguiu prever de forma satisfatória a temperatura na massa de grãos apresentando erros menores que $6,3 \%$.

Além disso, a modelagem proposta, aliada a um modelo de isoterma de equilíbrio, no caso o modelo proposto por Finkler et al. (2013), conseguiu prever a umidade na massa de grãos em função da posição e do tempo mesmo sem ter considerado inicialmente a convecção natural, a migração de umidade e a geração de calor. As maiores variações de umidade verificadas foram obtidas próximas à parede do silo onde se deram as maiores variações de temperatura.

\section{NOMENCLATURA}

$\mathrm{Cp}_{\text {silo }} \quad$ Calor específico do silo, $\mathrm{J} \cdot \mathrm{kg}^{-1} \cdot \mathrm{K}^{-1}$

$\mathrm{e}$ Espessura da parede do silo, $\mathrm{m}$

E\% Erro absoluto médio relativo percentual

ET Equação do tempo ("Ephemeris 
Transit")

$\mathrm{h}_{\mathrm{ext}} \quad$ Coeficiente de troca térmica na parte externa do silo, W. $\mathrm{m}^{-2} \cdot \mathrm{K}^{-1}$

$\mathrm{h}_{\text {int }} \quad$ Coeficiente de troca térmica na parte interna do silo, $\mathrm{W} \cdot \mathrm{m}^{-2} \cdot \mathrm{K}^{-1}$

$\mathrm{H} \quad$ Altura do silo, $\mathrm{m}$

$\mathrm{H}_{\mathrm{L}} \quad$ Hora legal, $\mathrm{h}$

$\mathrm{H}_{\mathrm{M}} \quad$ Ângulo horário do Sol médio, $\mathrm{h}$

$\mathrm{H}_{\mathrm{V}}$ Ângulo horário do Sol verdadeiro, $h$

$\mathrm{q}_{\mathrm{dif}} \quad$ Densidade de fluxo de energia de radiação difusa, $\mathrm{W} \cdot \mathrm{m}^{-2}$

$\mathrm{q}_{\mathrm{H}} \quad$ Densidade de fluxo de energia de radiação solar direta e difusa em uma superfície horizontal, W. $\mathrm{m}^{-2}$

$\mathrm{q}_{\mathrm{H}}^{\text {dir }}$ Densidade de fluxo de energia de radiação solar direta em uma superfície horizontal, W. $\mathrm{m}^{-2}$

$\mathrm{q}_{\mathrm{rad}}^{\text {lat }}$ Densidade de fluxo de energia de radiação solar (direta e difusa) absorvida pela parede lateral do silo, W.m ${ }^{-2}$

$\mathrm{q}_{\mathrm{rad}}{ }^{\text {topo }}$ Densidade de fluxo de energia de radiação solar (direta e difusa) absorvida pelo topo do silo, W. $\mathrm{m}^{-2}$

qv Densidade de fluxo de energia de radiação solar direta e difusa em uma superfície vertical voltada para o Sol, W.m ${ }^{-2}$

$\mathrm{qv}^{\text {dir }}$ Densidade de fluxo de energia de radiação solar direta em uma superfície vertical voltada para o Sol, W.m ${ }^{-2}$

$\mathrm{R} \quad$ Raio interno do silo, $\mathrm{m}$

t Tempo, s

T Temperatura, $\mathrm{K}$

$\mathrm{T}_{\mathrm{amb}} \quad$ Temperatura do ar, $\mathrm{K}$

$\mathrm{T}_{\mathrm{P}} \quad$ Temperatura da parede do silo, $\mathrm{K}$

$\mathrm{T}_{\text {solo }}$ Temperatura do solo abaixo do silo, $\mathrm{K}$

UR Umidade relativa decimal (p/p)

$\mathrm{X}_{\mathrm{e}} \quad$ Umidade de equilíbrio (\%b.s.)

$\mathrm{z} \quad$ Coordenada axial, $\mathrm{m}$

zen Distância zenital, graus

$\alpha \quad$ Absortividade do silo

$\alpha_{\text {lat }} \quad$ Porcentagem da área lateral atingida pela radiação solar

$\alpha_{\mathrm{T}} \quad$ Difusividade térmica efetiva, $\mathrm{m}^{2} \cdot \mathrm{s}^{-1}$

$\delta \quad$ Declinação solar, graus

$\varepsilon \quad$ Emissividade do silo

$\phi \quad$ Latitude, graus

$\rho_{\text {silo }} \quad$ Massa específica do silo, kg.m ${ }^{-3}$ $\sigma$ constante de Stefan-Boltzmann, $5,67 \cdot 10^{-8} \mathrm{~W} \cdot \mathrm{m}^{-2} \cdot \mathrm{K}^{-4}$

\section{AGRADECIMENTOS}

Agradecemos a Fundação de Amparo à Pesquisa do Estado de São Paulo (FAPESP), o Programa Nacional de Excelência (PRONEX/FINEP) e o Conselho Nacional de Desenvolvimento Científico e Tecnológico (CNPq) pelo auxílio financeiro. Agradecemos também ao professor Basílio Santiago (IF-UFRGS) por seus esclarecimentos em Astrometria e ao Dr. João Luiz Kohl Moreira do Observatório Nacional (ON) pela indicação do endereço eletrônico para a obtenção da "Ephemeris Transit".

\section{REFERÊNCIAS}

ALAGUSUNDARAM, K., JAYAS, D. S., WHITE, N. D. G. \& MUIR, W. E. 1990. Three Dimension, Finite Element, Heat Transfer Model of Temperature Distribution in Grain Storage Bins. Transactions of the ASAE, 33, 577-584.

ANDRADE, E. T., COUTO, S. M., QUEIROZ, D. M., FARONI, L. R. D. \& DAMASCENO, G. S. 2004. Simulação Tridimensional da Distribuição de Temperatura em Milho Armazenado em Silo Cilíndrico. Revista Brasileira de Armazenamento, 29, 1-12.

BASUNIA, M. A \& ABE T. 1999. Simulation of Temperature and Moisture Changes During Storage of Rough Rice in Cylindrical Bins Owing to Weather Variability. Journal of Agricultural Engineering Research, 65, 223-233.

CARVALHO, N. M. \& NAKAGAWA, J. 1980. Sementes: Ciência, Tecnologia e Produção, Campinas, Fundação Cargill.

CHRISTENSEN et al. (1969) apud MUIR, W. E. 1970. Temperature in Grain Bins. Canadian Agricultural Engineering, 12, 21-24.

CONVERSE, H. H., GRAVE, A. H. \& CHUNG, D. S. 1973. Transient Heat 
Transfer Within Wheat Stored in a Cylindrical Bin. Transactions of the ASAE, 16, 129-133.

COOPER, P. I. 1969. The Absorption of Solar Radiation in Solar Stills. Solar Energy, 12, 333-346.

DONA, C. L. G. \& STEWART JR, W. E. 1988. Numerical Analysis of Natural Convection Heat Transfer in Stored High Moisture Corn. Journal of Agricultural Engineering Research, 40, 275-284.

DUFFIE, J. A \& BECKMAN, W. A. 1974. Solar Energy Thermal Processes, New York, John Wiley and Sons.

EMBRAPA. 2005 Dados da estação meteorológica da Empraba de CampinasSP. Disponível em: $<$ http://www.cnpm.embrapa.br/projetos/es tacaometeorologica/estacao.php> Acesso: 01 dez. 2005.

FILHO, K. S. O. \& SARAIVA, M. F. O. 2004. Astronomia e Astrofísica, Porto Alegre, Editora Livraria da Física.

FINKLER, A. T. J., PALÚ, F., SILVA, E. A. S. \& MOREIRA, M. F. P. Análise das Isotermas de Equilíbrio de Adsorção e Dessorção da Soja. XXXVI Congresso Brasileiro de Sistemas Particulados (XXXVI ENEMP) 2013 Maceió, Brasil.

FOHR, J. P. \& MOUSSA, H. B. 1994. Heat and Mass Transfer in a Cylindrical Grain Silo Submitted to a Periodical Wall Heat-flux. Internatinal Journal of Heat and Mass Transfer, 37, 1699-1712.

HASSE, G. 1996. O Brasil da Soja Abrindo Fronteira, Semeando Cidades, Porto Alegre, L\&PM Editores S. A.

HOLMAN, J. P. 1983. Transferência de Calor, São Paulo, McGraw-Hill.

IGUAZ, A., ARROQUI, C., ESNOZ, A., VÍRSEDA, P. 2004. Modelling and Validation of Heat Transfer in Stored Rough Rice without Aeration. Biosystems Engineering, 88, 429-439.
JIA, C., SUN, D. \& CAO, C. 2000a. Mathematical Simulation of Temperature Fields in a Stored Grain Bin due to Internal Heat Generation. Journal of Food Engineering, 43, 227-233.

JIA, C., SUN, D. \& CAO, C. 2000b. Finite Element Prediction of Transient Temperature Distribution in a Grain Storage Bin. Journal of Agricultural Engineering Research, 76, 323-330.

JIA, C., SUN, D. \& CAO, C. 2001. Computer Simulation of Temperature Changes in a Wheat Storage Bin. Journal of Stored Products Research, 37, 165-177.

JIAN, F., JAYAS, D.S., WHITE, N.D.G., ALAGUSUNDARAM, K. 2005. A ThreeDimensional, Asymmetric, and Transient Model to Predict Grain Temperatures in Grain Storage Bins. Transactions of the ASAE, 48, 263-271.

JIAN, F., JAYAS, D. S. \& WHITE, N. D. G. 2009. Temperature Fluctuations and Moisture Migration in Wheat Stored for 15 Months in a Metal Silo in Canada. Journal of Stored Products Research, 45, 82-90.

KREITH, F. 1973. Princípios da Transmissão de Calor, São Paulo, Edgard Blücher LTDA.

LORINI, I., MIIKE, L. H. \& SCUSSEL, V. M. 2002. Armazenagem de Grãos, Campinas, Instituto Bio Geneziz (IBG).

MOREIRA, M. F. P. \& FREIRE, J. T. Previsão da Temperatura em Silos Verticais Contendo Soja. Parte I: Determinação das Propriedades Térmicas da Soja. XXXI Congresso Brasileiro de Sistemas Particulados (XXXI ENEMP) 2004 Uberlândia, Brasil.

MOSHIER. 2005. Programa computacional "aa-56" para a obtenção do valor de ET. Disponível em: <http://www.moshier.net/> Acesso: 01 dez. 2005.

MUIR, W. E. 1970. Temperature in Grain Bins. Canadian Agricultural Engineering, 
$12,21-24$.

PRACHAYAWARAKORN, CHOTEBOON, C. SOPONROIINARIT, S. 2005.
Simultaneous Momentum, Heat, and Mass Simultaneous Momentum, Heat, and Mass
Transfer with Color Change during Paddy Storage in Silo. Drying Technology, 23, 205-223.

PUZZI, D. 1986. Abastecimento $e$ armazenagem de grãos, Campinas, Instituto Campineiro de Ensino Agrícola.

RODRÍGUEZ, M. C., GONZÁLEZ, G. M. M., BOLAÑOS, J. L. N., ÁLVAREZ, J. E. B., MARTÍNEZ, R. R. \& ISLAS, H. J. 2011. Transient Numerical Study of the Effect of Ambient Temperature on 2-D Cereal Grain Storage in Cylindrical Silos. Journal of Stored Products Research, 47, 106-122.

RUSINEK, R. \& KOBILKA, R. 2014. Experimental Study and Discrete Element Method Modeling of Temperature Distributions in Rapeseed Stored in a Model Bin. Journal of Stored Products Research, http://dx.doi.org/10.1016/j.jspr.2014.03.00 9

SILVA, W. R. 1998. Seed Performance After Exposure to High Temperatures. Scientia Agricola, 55, 102-109.

SMITH, E. A. \& SOKHANSANJ, S. 1990a. Moisture Transport Caused by Natural Convection in Grain Stores. Journal of Agricultural Engineering Research, 47, 23-34.

SMITH, E. A.; SOKHANSANJ, S. 1990 b. Natural Convection and the Temperature of Stored Produce - A Theoretical Analysis. Canadian Agricultural Engineering, 32, 91-97

STENNING, B. C. 2012. Modelling and Simulation Heat Transfer in Wheat Stored in a Simulated Sealed Pit, International Journal of Food Engineering, 8, Article 23, 16p. DOI: 10.1515/1556-3758.1308
YACIUK, G., MUIR, W. E. \& SINHA, R. N. 1975. A Simulation Model of S., Temperature in Stored Grain. Journal of \& Agricultural Engineering Research, 20, 245-258. 